\title{
"The library catalog is definitely the best place to find articles!" Overconfidence among Undergraduate Library Users
}

\author{
Katelyn Angell \\ Assistant Professor/First Year Success Librarian \\ Brooklyn Campus Library \\ Long Island University \\ Katelyn.Angell@liu.edu \\ Dr. Gary Kose \\ Professor \\ Psychology Department \\ Brooklyn Campus \\ Long Island University \\ gkose@msn.com
}

\section{Abstract}

Beginning in the 1980s, researchers in a wide array of academic disciplines surveyed undergraduate students to learn if they were overconfident with regard to their knowledge of disciplinary subject matter. The majority of researchers have found that students tend to overestimate their abilities within a certain body of knowledge. Up until recently, most of these projects were conducted in the fields of education, psychology, and economics. In the past few years, several academic librarians have applied these methodologies to their undergraduate patrons, curious as to whether this population demonstrates similar overconfidence in their grasp of academic research. The present study surveyed 34 undergraduates enrolled in psychology classes at a large urban university in the United States to determine if these students were overconfident about their knowledge in key library research areas. These questions included citing and referencing, identifying components of scholarly and popular sources, and developing and applying searches in electronic resources. A general knowledge survey was created for comparison purposes. Statistical analysis showed that students displayed marked overconfidence on both tools, signifying a need for increased library and metacognitive skill instruction.

\section{Keywords}

Overconfidence, psychology, information literacy, research skills, university students 


\section{Introduction}

A sound belief in one's ability to accomplish is an indispensable ingredient in the recipe for success. In order to succeed in higher education it is paramount for students to possess self-confidence, especially those new to a challenging university environment. However, a diverse and steadily growing body of literature argues that too much confidence, frequently referred to as "overconfidence," can negatively impact academic performance. Overconfidence can complicate and stymie academic achievement because its presence causes students to overestimate the depth and breadth of their knowledge on specific skills or content.

Overconfident learners are vulnerable to misconceptions of self and a sense of entitlement. This construct has been explored in detail by researchers within sciences and social sciences disciplines. Twenge, Campbell, and Gentile (2012) conducted extensive research on generational differences between university students on a host of self-evaluation variables, including intellectual self-confidence. Students surveyed in 2009 defined themselves as intellectually above average a startling $54 \%$ more than students measured in 1966.

Despite the fact that learning university level library skills is a cornerstone of academic success, very little research on overconfidence has been conducted in library and information science (LIS). If students are overly confident about their grasp of research skills, this overconfidence could result in a chasm between imagined and real knowledge. This could potentially lead to inaccurate information selection, evaluation, usage, and citing practices within myriad disciplines.

\section{Literature Review}

Definitions of "overconfidence" differ among academic disciplines, but within psychology overconfidence is best described as "a judgmental error in which people overestimate their own accuracy. Specifically, overconfidence is defined as a positive difference between confidence and accuracy" (Schaefer, Williams, Goodie, \& Campbell, 2004, p. 473). Studies investigating overconfidence among university students encompass a wide range of academic subjects in addition to psychology, particularly economics (Nowell \& Alston, 2007). Presently, only a few studies measure overconfidence levels within an academic library setting (Gross \& Latham, 2012; Gustavson \& Nall, 2011; Molteni \& Chan, 2015; Polkinghorne \& Wilton, 2010). While the majority of research on overconfidence has found a relationship with incorrect answers on tests, not all studies published by librarians have found these results. The present study will build upon these studies examining library research skills. Two research questions will be addressed. First, is there a relationship between overconfidence and incorrect answers on an academic library skills test? Second, if this relationship exists, is it limited to library skills or does it extend to other subject areas?

Researchers have been studying the presence and influence of overconfidence on academic tasks since the 1980s. One of the earliest available papers on this topic is a 
report written by Zechmeister, Rusch, and Markell (1983). Their primary objective was to assist students in increasing their metacognitive confidence about judgment capabilities. Groups of high and low performing students completed pretests and posttests comprised of general knowledge questions. Half of the students in each achievement group received an intervention which trained them to better separate known and unknown information, and half of the students were required to explain their rationale for selecting their answers. The findings showed that low achievers experienced greater overconfidence than high achievers, results that would be repeated in future studies. Additionally, both groups benefited from confidence judgment training, and the need to justify answer selection on the testing instrument decreased overconfidence for low achievers. Students who believe too strongly in the automatic correctness of a first guess and fail to critically consider other options place themselves in a compromising academic position.

In general, studies investigating overconfidence demonstrate that low performing students are more disposed to overestimate their academic achievement than high performing students. Hacker, Bol, Horgan, and Rakow (2000) investigated the predictive abilities of 99 psychology students on three multiple choice examinations before (prediction) and after they completed tests (postdiction). Results showed that students who received high marks were very accurate in their predictions and postdictions of test performance. In contrast, the predictions of lower academically performing students exhibited marked overconfidence: as grades fell, overconfidence levels increased. The majority of students displayed fair accuracy in the postdictions, save those with the poorest academic performance. These findings illustrate the potential of overconfidence to cause a lack of awareness of one's own knowledge deficits. Rather than confront and resolve this lack, a person runs the risk of displacing their low grades upon external factors, such as difficult examinations or academically rigorous professors.

Most of the studies examining student confidence with regard to library research skills pertain to helping university students build confidence by improving their information finding skill sets (Gilbert, 2009; Jacoby \& O'Brien, 2005; Roselle, 2009). In contrast, the research in psychology and education has documented the problematic aspects of overconfidence. Given the high percentage of undergraduate students that visit the library for research instruction, there should be more examinations of the effects of overconfidence within the library environment.

An additional justification for the need of articles on this topic is the fact that multiple LIS studies have demonstrated a positive association between library use and academic achievement. Chiteng Kot and Jones (2015) found that first semester undergraduates who attended library instruction sessions received a higher term GPA than students in a control group without instruction. In addition to formal library sessions, research shows that student use of library resources can contribute to academic success. Cherry, Rollins, and Evans (2013) learned that undergraduate students with higher GPAs utilized electronic library resources such as online databases and e- journals more frequently than students with lower GPAs. This study was not a true experiment, but it 
did generate a correlation suggesting that usage of electronic library resources is one of many important factors associated with academic achievement.

The mastery of academic research skills can greatly benefit students in their future career choices. For example, the ability to locate and evaluate high-quality information is a significant responsibility of many health care professionals. Academic and medical librarians can teach students the skills they will need to succeed in careers as doctors and nurses, such as how to use the popular databases PubMed and CINAHL (Prusin, 2012). Clarifying the significance of this work, Prusin writes, "With patient safety involved, the importance of teaching these students advanced search strategies cannot be overstated" (p. 10).

At present, only a few studies within LIS literature discuss the important topic of overconfidence in academic library patrons. Polkinghorne and Wilton (2010) taught a political science research methods course and learned that student beliefs in the proficiency of their research skills did not necessarily translate into strong skills. For example, highly confident students frequently exhibited problems with citing, searching, and building research statements. Two causes of overconfidence within library research were proposed: a lack of personal interest in research methods, and anxiety surrounding the research process (p. 460). It was noted that in addition to augmenting information literacy skills they hoped their activity-based agenda could help decrease overconfidence. One explanation for this decline could be that an authentic increase in research knowledge would curb the need for an inflated sense of confidence.

Gross and Latham (2012) conducted research to determine if first year university students were overconfident about their information literacy knowledge. Participants from two community colleges were administered an information literacy skills test. All were given a survey before and after the test which required them to predict their score and how it compared with the marks of fellow participants. Nearly all students scored in the below-proficient range. On average, students greatly overestimated their scores on the pretests and posttests. Similarly, students in both groups were very confident that they had outperformed their peers. However, their estimates turned out to be noticeably higher than their true scores. The authors posit several steps librarians can take to help students increase their information literacy skills, such as to develop information literacy diagnostic tools and to not overestimate that students are technologically competent.

Gustavson and Nall (2011) published an article which investigated this very topic. They posed two research questions: (a) What is the correlation between self-reported confidence levels and tested skills? (b) What is the relationship between age and high school grades on confidence level and skill? First year students at a large university served as participants. All were given a combination overconfidence survey and library research skills test. Students ranked their confidence on a Likert scale ranging from one (not confident) to five (very confident). The skills test consisted of eight multiple choice questions on important academic research topics. In contrast to similar studies, the findings did not show a statistically significant difference between confidence level 
and score on library skills. Only around $20 \%$ of respondents reported high confidence in their library research capabilities.

Students with the highest scores on the skills test were rather modest about their abilities (as the group who rated their confidence as three showed the best performance). Participants with responses of four or five were identified as overconfident. Forty-four (12\%) of the participants were frank about their unfamiliarity with library research, identifying as "not confident." This honesty was reflected in the average scores of the skills test (students rating their confidence as a one overall received the lowest scores). These results suggest that students who place themselves in the middle of the confidence spectrum are realistic about their knowledge, displaying an appropriate amount of certainty of their mastery. The correlation between test scores and confidence levels was very low (.12), meaning that the researchers did not find a significant relationship between these two variables.

Additionally, Gustavson and Nall (2011) explored four other factors to learn if they elicited overconfidence: age, gender, high school grades, and prior library instruction. Only one-on-one research assistance from a librarian was associated with an overconfident participant. A few strategies for reducing overconfidence were suggested such as using interactive teaching to help students, and beginning library instruction sessions with a quiz focusing on assessing student knowledge and demystifying popular false beliefs (p. 303).

Most recently, Molteni and Chan (2015) investigated information literacy skills and confidence levels of health sciences students. Over 200 students completed a quiz focusing on research within the health sciences. The quiz consisted of demographic questions and four information literacy tasks. In the final part participants answered multiple choice questions about library research, such as defining primary research and identifying an appropriate database for a given topic.

The results showed a rather high percentage of incorrect or unsure answers, ranging from $38 \%$ for revising a databases search to $49 \%$ for scholarly versus popular sources. Participants who exhibited high levels of confidence in their skills had more correct answers than those with less confidence, but for some tasks very confident students were as likely to get an answer wrong. The authors concluded, "Confidence does not appear to be a reliable indicator of competence" (Molteni \& Chan, 2015, p. 5).. In other words, just because a student is self-assured in their abilities does not necessarily mean that they are adept researchers.

In the present study, undergraduate students were given questionnaires assessing their library research skills and general knowledge. The participants provided an answer to each test question as well as a rating of their confidence in their choice. The null hypothesis predicted that there would not be a significant relationship between test scores and confidence level on either instrument. The alternative hypothesis predicted that the highest levels of confidence would be found in participants with the most 
incorrect answers. Additionally, differences between the two types of information were investigated.

\section{Method}

\section{Participants}

Thirty-four undergraduate students served as a convenience sample for this project. These participants ranged in age from 19 to 56 years old ( $M=25$ years, $S D=7.95$ years). There were 31 women and three men. The majority of students were fourth years (13) or third years (eight).

Fifteen participants reported that they had visited the library at least once with a class for formal library instruction. Eight participants indicated that they had never visited the library for instruction, and eleven participants did not answer this question.

\section{Measures}

A questionnaire was developed for this study that assessed library research skills. The Library Knowledge Confidence Scale (see Appendix A) consisted of ten true/false questions pertaining to three fundamental research skills: citing and referencing, identifying components of scholarly and popular sources, and developing and applying searches in electronic resources. After circling an answer the participant next noted how confident they were of their response.

Confidence level was indicated by way of a confidence scale ranging from $50 \%$ (just guessing) to $100 \%$ (absolutely sure). Overconfidence was operationalized as a confidence score of 90 or $100 \%$ on an incorrect answer. For statistical analysis purposes confidence levels were changed to a Likert scale ranging from one (low) to three (high). Confidence levels of $50-60 \%$ were recoded as one, levels of $70-80 \%$ were recoded as two, and levels of $90-100 \%$ were recoded as three (overconfident).

A second questionnaire was developed that assessed general knowledge. The General Knowledge Confidence Scale consisted of ten true/false questions (see Appendix B). Several of the questions on the instrument were adapted from the General Knowledge Questionnaire devised by Michailova (2010). The test taking instructions for the general knowledge survey were identical to the directions listed on the library skills survey.

\section{Procedure}

The Library Knowledge Confidence Scale and the General Knowledge Confidence Scale were distributed to participants during class. Students completed the instruments and provided anonymous basic demographic information. Once the scales were collected from participants the scales were coded from one to 34 and then graded on a scale from one to 100, with each question on the two scales worth ten points. Any answer left blank was marked incorrect. 


\section{Results}

The mean and standard deviations of the overall test scores and confidence levels for the library skills and general tests are presented in Table 1 . Scores were relatively low on both tests ( $M=60$ on the library scale and $M=69$ on the general knowledge scale). In contrast, confidence levels were high $(M=2.35$ on the library skills scale and $M=2.37$ on the general scale). A Pearson correlation revealed a moderate positive relationship between scores on the library skills and general skills tests $(r=.36, p<.05)$, indicating that performance on the two tests was similar.

Table 1. Means and standard deviations for the Library Knowledge and General Knowledge scales and Confidence Levels.

\begin{tabular}{lllll}
\hline & \multicolumn{2}{l}{ Scale } & \multicolumn{3}{l}{ Confidence } \\
& $M$ & $(\mathrm{sd})$ & $\mathrm{M}$ & $(\mathrm{sd})$ \\
Library Skills & 60.9 & $(15.47)$ & 2.35 & $(.35)$ \\
General Knowledge & 69.41 & $(20.74)$ & 2.37 & $(.47)$ \\
\hline
\end{tabular}

In order to examine the differences between the confidence levels and the number of incorrect responses on the scales, each participant was categorized as low, medium, and high confidence. If seven of the ten questions on the library knowledge scale were rated high confidence (three), the participant was categorized as high confident; if seven of the ten questions were rated low confidence (one), the participant was categorized as low confident; if seven of the ten questions were rated intermediate confidence (two), then the participant was categorized as medium confident. This same procedure was followed for the general knowledge scale. Thus, each participant had four scores: a Library Skills Score (number of incorrect answers), a Library Skills Confidence Category (low, medium, and high), a General Knowledge Score (number of incorrect answers), and General Knowledge Category (low, medium, and high).

The differences between the confidence categories on the number of incorrect answers on the scale scores were examined by computing two one-way between-group ANOVAs for library skills and general knowledge, with confidence category (low, medium, and high) as the independent variable and the number of incorrect answers on scale score as the dependent variable. The means and standard deviations for the number of incorrect responses on the library test are presented in Table 2. 
Table 2. Means and standard deviations for the number of incorrect answers on the Library Knowledge and General Knowledge scales presented by Confidence Category.

\begin{tabular}{|c|c|c|c|}
\hline & \multicolumn{3}{|c|}{ Confidence Category } \\
\hline & $\begin{array}{l}\text { Low }(n=7) \\
M \quad(\mathrm{sd})\end{array}$ & $\begin{array}{l}\text { Medium }(n=18) \\
M \quad(\mathrm{sd})\end{array}$ & $\begin{array}{l}\text { High }(n=9) \\
\underline{M} \quad(\mathrm{sd})\end{array}$ \\
\hline \multirow{3}{*}{$\begin{array}{l}\text { Library Skills } \\
\text { Score }\end{array}$} & 48.57 (19.51) & $60.00(12.36)$ & $70.00(12.24)$ \\
\hline & \multicolumn{3}{|c|}{ Confidence Category } \\
\hline & $\begin{array}{l}\operatorname{Low}(\mathrm{n}=4) \\
\mathrm{M} \quad(\mathrm{sd})\end{array}$ & $\begin{array}{l}\text { Medium }(n=16) \\
M \quad(s d)\end{array}$ & $\begin{array}{l}\text { High }(n=14) \\
M \quad(s d)\end{array}$ \\
\hline General Score & 55.00 (31.09) & $65.00(17.12)$ & 78.57 (20.73) \\
\hline
\end{tabular}

The ANOVA revealed a significant effect for confidence category $(F(2,31)=4.61, p=$ $.02, d=.23$ ). Post hoc (Bonferroni) analyses showed that there were significantly fewer incorrect responses in the low confident category $(M=48.57)$ than in the high confident category $(M=70.00)(p=.02)$.

The means and standard deviations for the number of incorrect responses to the general scale are also presented on the bottom of Table 2. The ANOVA revealed a marginal effect for confidence category $(F(2,31)=3.03, p=.06, d=.16)$. As can be seen in Table 2, again there were fewer incorrect responses in the low confident category $(M=55.00)$ than in the high confident category $(M=78.57)$. No doubt, the marginal effect was influenced by the small number of participants in the low confidence category. 


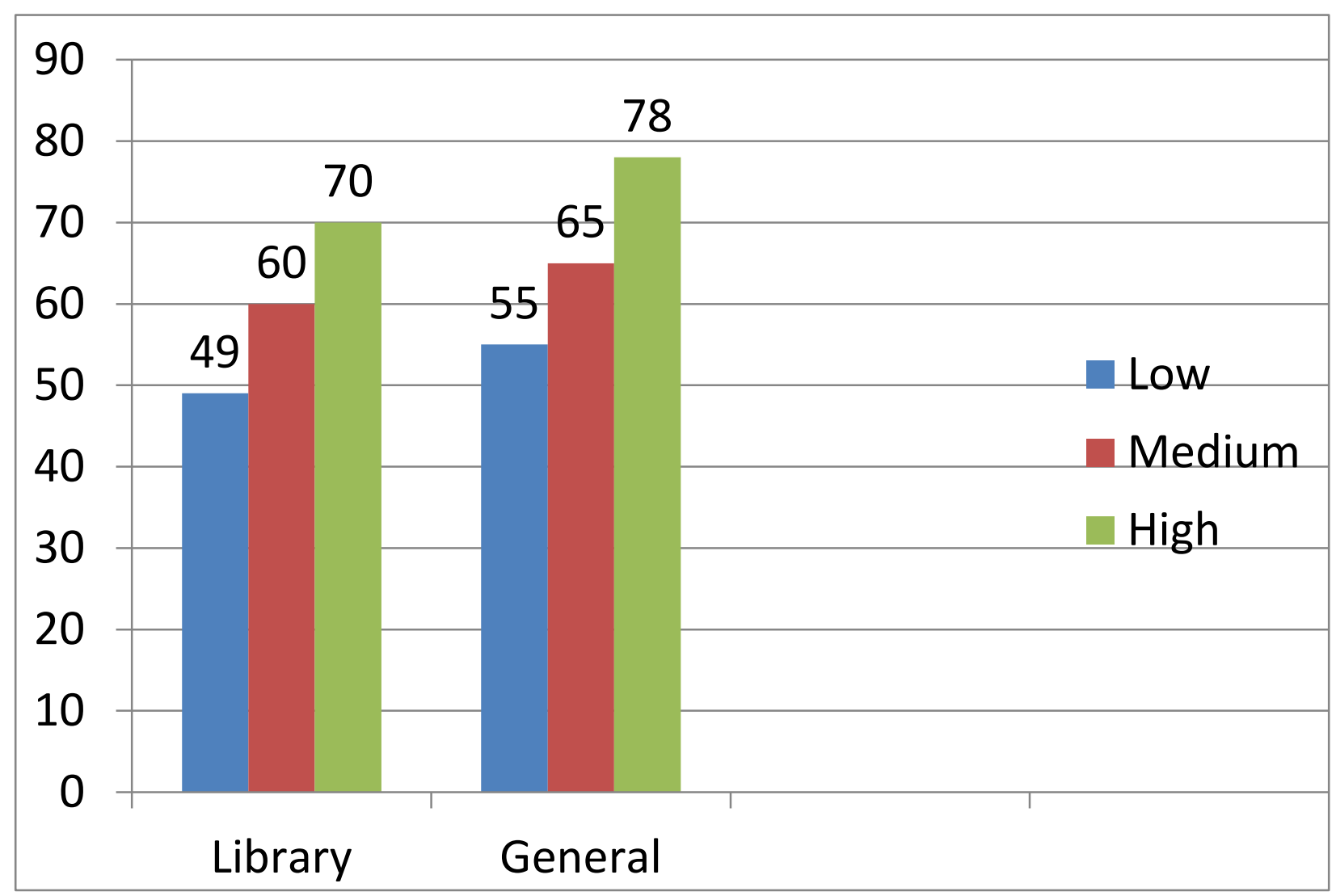

Figure 1. Mean number of incorrect responses presented by Confidence Category.

Thus, the findings showed a clear overconfidence effect, which can be seen in Figure 1. The alternative hypothesis was confirmed. For library skills, there were significantly fewer incorrect responses among the low confidence group compared to the high confidence group; to a lesser extent, the same pattern of results were found for the general knowledge scale.

\section{Discussion}

Similar to the majority of psychological and educational literature researching overconfidence, the data from the present study suggests overconfidence within college students assigned library skills tasks. The 34 study participants received relatively low scores on both the library skills and general knowledge tests. Overall, they received more wrong answers on the general test (198) than on the library test (179). The scores appear especially low when translated into the standard A-F grading system. Table 1 reports that the mean and median of the library skills test were around 60 out of 100 (D), with scores ranging from $20(\mathrm{~F})$ to $90(\mathrm{~A})$. The mean of the general skills test was around $69(D)$, with scores ranging from ten $(F)$ to $100(A)$.

A similar lack of research preparedness among undergraduate library patrons was found by Gross and Latham (2012). Library skills tests were administered to groups of first year students at two universities. The majority of students scored in the below- 
proficient range, with a mean of 44 out of 100 at one school and a slightly better average of 53 at the other institution. Although the average scores at both universities translate into failing grades, the participants nonetheless displayed noticeable overconfidence with regard to their library skills. A particularly salient finding is that the least competent students were most vulnerable to failing to properly calibrate self-views and research skills.

Despite the low scores on both scales in the current study, results showed that students were often overly certain about their answer choices. Figure 1 indicates that out of the three confidence categories, low, medium, and high, the largest number of incorrect answers fell into the high category for the library (70) and general (78) skills tests. In contrast, the smallest number of incorrect answers appeared in the low confidence column, with 49 incorrect on the library test and 55 on the general scale. The mean confidence level for the library test was 2.35 , and it was 2.37 for the general test, meaning that average confidence registered between medium and high. These figures indicate that participants were more likely to assign a high confidence level rather than a low or medium level to an incorrect answer on both tests. An ANOVA showed a very significant relationship between confidence level and incorrect answers on the library skills scale, confirming the alternative hypothesis for the first research question presented in the study. These results reflect the conclusions of academic library researchers Gross and Latham (2012) and Polkinghorne and Wilton (2010).

The strikingly low scores on library skills measures recorded by Gross and Latham (2012) and the present authors suggest that the general undergraduate population could benefit from additional instruction in university level research from a librarian. Correspondingly, the political science students in Polkinghorne and Wilton's (2010) study demonstrated ample confidence in their academic research skills, although the researchers noted "in-course evidence of skill deficiencies in these areas, such as weak citation practices, sloppy search techniques, and problems constructing research questions and theses" (p. 460). This disconnect between student beliefs and reality could be the product of a lack of interest, an excess of academic anxiety, and/or a lack of qualified preparation.

A dearth of formal library training in primary and secondary school is not uncommon; students are often underprepared for university research. Inadequate exposure to library instruction could spur students to either go it alone or consult a friend for advice, potentially resulting in the development of flawed research skills. Overconfidence and incompetence can be the outcome, in which students might not even be able to recognize that they are underproficient in a skill area. An analysis of the research skill sets of health sciences undergraduates by Molteni and Chan (2015) corroborated that confidence level was not a reliable indicator of proficiency. Gross and Latham (2012) conclude that "incompetence is best overcome through the development of knowledge and skill" (p. 581).

However, it is important to note that not all other studies on this topic within academic libraries have shown marked overconfidence among students. Gustavson and Nall 
(2011) also presented undergraduate participants with a test that measured a range of basic academic research skills. They calculated a very weak correlation between test scores and confidence ( $p=.12)$, signifying that overall there was not a significant relationship between these two variables.

Academic librarians must collaboratively develop strategies to decrease overconfidence and increase research skills. One idea for bettering these outcomes is partnering with teaching faculty to develop reflective and interactive courses (Polkinghorne \& Wilton, 2010 , p. 471) featuring an embedded librarian. Another course of action is to investigate self-assessment of skills in disciplines outside of LIS, such as "human information behavior, the development of user interfaces, and evaluation of information systems and services" (Gross \& Latham, 2012, p. 582). A final strategy could be to teach students to carefully evaluate each potential answer on a test. They could reflect on their reasons for deciding that an answer is correct, first writing their rationale and then discussing their thoughts with classmates and instructors. This process-oriented approach could easily be incorporated into the library classroom.

No matter the root cause, overconfidence is not limited to collegiate pursuits, as shown by the general skills test scores. There were more total incorrect answers in all three confidence levels on the general test than on the library test, and the ANOVA reported a minimal relationship between confidence level and test performance. Incorporated into the project for comparison purposes, the general knowledge scale provided a unique means of learning if students display overconfidence in multiple subject areas.

Therefore, among this sample overconfidence was not limited to research skills; the tendency to overinflate one's knowledge can be extrapolated to additional topical areas. This confirms the alternative hypothesis for the second research question, which was concerned with determining if overconfidence extended to non-library related tasks. The real problem here is not necessarily a content issue; correct answers can easily be learned. Rather, the true obstacle is teaching people to unlearn this trend toward overconfidence, and thus help them to improve their academic performance.

\section{Limits and Future Directions}

Only a few researchers have applied tactics garnered from overconfidence literature to an academic library setting. These studies have yielded conflicting results, with some determining overconfidence and others not finding this association. The objective of the present study was to contribute to this small but compelling body of knowledge, with an added goal of providing future researchers with successes and drawbacks of the methods and research design.

There were several limitations present within this study. First, the lack of diversity among participants in terms of gender and class year spurred the researcher not to include these variables within statistical analyses. Out of 34 participants only three identified as men, figures which would not provide a balanced assessment of potential gender differences. Similarly, the majority of participants were third or fourth years. A 
future study could recruit a more divergent group of participants in terms of both gender and class year. Nowell and Alston (2007) found that men were more likely than women to exhibit overconfidence on their grades in an economics class. Given this difference between male and female undergraduates on an economics assessment it would be interesting to more thoroughly explore library skills through the lens of gender.

A related drawback is a lack of variance among the class year of participants. The fact that so many students in the present study were in their third or fourth year of university could have had a significant effect on the study's results. For example, students with several years of university research under their belts could feasibly have a better grasp of what they do and do not know, as opposed to students brand new to university.

There are multiple research designs which can be employed in subsequent studies on overconfidence within undergraduate library users. A future study on this topic could be constructed as a true experiment, randomly assigning students to three different conditions related to library skills. One condition would focus on citing and referencing, another on generating keywords and search strings, and the final condition would focus on identifying and using appropriate electronic resources. All participants would be provided with a confidence scale, and data analysis could determine if there were a meaningful difference between skill and confidence level among the three groups.

Additionally, a longitudinal study would be an excellent means of measuring confidence levels of academic library patrons during their entire university careers. This method would allow researchers to make important observations about confidence and research skill level over the course of four years at both the individual and group level. Librarians could use this data to inform and structure their library session planning, tailoring key skill developments to specific class years.

In conclusion, students enrolled in psychology classes at the university under consideration were not overly adept at distinguishing between knowledge they did and did not possess. This finding was apparent not only in relation to library research skills, but also translated to a comparative tool comprised of general trivia questions. The markedly low scores on the library test, $60 \%$ on average, signify that the sample has not yet mastered basic research skills integral to academic success. Despite these low scores, students exhibited definitive overconfidence pertaining to their mastery of academic research skills. Parallel findings have appeared in at least three other LIS studies, indicating to both librarians and teaching faculty that undergraduates could truly profit from more library instruction than they currently receive. Such instruction should incorporate collaborative activities and reflective exercises intended to sharpen metacognitive and critical thinking skills, with an emphasis on improving self-monitoring abilities (Molteni \& Chan, 2015). Through these processes librarians can help students to better locate, evaluate, and apply high-quality information with confidence, not overconfidence. 


\section{References}

Bruine de Bruin, W., Parker, A.M., \& Fischhoff, B. (2007). Individual differences in adult decision-making competence. Journal of Personality and Social Psychology, 92(5), 938-956.

Cherry, E., Rollins, S.H., \& Evans, T. (2013). Proving our worth: The impact of electronic resource usage on academic achievement. College \& Undergraduate Libraries, 20(3/4), 386-398.

Chiteng Kot, F., \& Jones, J.L. (2015). The impact of library resource utilization on undergraduate students' academic performance: A propensity score matching design. College \& Research Libraries, 76(5), 566-586.

Gilbert, J. K. (2009). Using assessment data to investigate library instruction for first year students. Communications in Information Literacy, 3(2), 181-192.

Gross, M., \& Latham, D. (2012). What's skill got to do with it?: Information literacy skills and self-views of ability among first-year university students. Journal of the American Society for Information Science and Technology, 63(3), 574-583.

Gustavson, A., \& Nall, H.C. (2011). Freshman overconfidence and library research skills: A troubling relationship? College \& Undergraduate Libraries, 18(4), 291306.

Hacker, D. J., Bol, L., Horgan, D. D., \& Rakow, E. A. (2000). Test prediction and performance in a classroom context. Journal of Educational Psychology, 92(1), 160-70.

Jacoby, J., \& O'Brien, N. P. (2005). Assessing the impact of reference services provided to undergraduate students. College \& Research Libraries, 66(4), 324-340.

Michailova, J. (2010). Development of the overconfidence measurement instrument for the economic experiment (Working Paper no. 34478).

Molteni, V.E., \& Chan, E.K. (2015). Student confidence/overconfidence in the research process. The Journal of Academic Librarianship, 41, 2-8.

Nowell, C., \& Alston, R. M. (2007). I thought I got an A! Overconfidence across the economics curriculum. Journal of Economic Education, 38(2), 131-142.

Polkinghorne, S., \& Wilton, S. (2010). Research is a verb: Exploring a new informationliteracy embedded undergraduate research methods course. The Canadian Journal of Information and Library Science, 34(4), 457-473. 
Prusin, T. (2012). Laboratory office hours as outreach in the health sciences: Better research skills for better careers. Public Services Quarterly, 8(1), 1-11.

Roselle, A. (2009). Preparing the underprepared: Current academic library practices in developmental education. College \& Research Libraries, 70(2), 142-156.

Schaefer, P.S., Williams, C.C., Goodie, A.S., \& Campbell, W.K. (2004). Overconfidence and the Big Five. Journal of Research in Personality, 38(5), 473-480.

Twenge, J. M., Campbell, W. K., \& Gentile, B. (2012). Generational increases in agentic self-evaluations among American university students, 1966-2009. Self \& Identity, 11(4), 409-427.

Zechmeister, E. B., Rusch, K.M, \& Markell, K.A. (1983). Training university students to assess accurately what they know and don't know (NIE-G-81-0093).

Washington, DC: National Institute of Education. 


\section{Appendix A: Library Knowledge Confidence Scale}

Age:

Gender:

Year in school:

Have you visited the library with a class for library instruction? Yes/No (Hint: These classes are usually held on the fifth floor of LLC)

This scale is based on the Adult Decision-Making Competence (A-DMC) scale. Citation: Bruine de Bruin, W., Parker, A.M., \& Fischhoff, B. (2007). Individual differences in adult decision-making competence. Journal of Personality and Social Psychology, 92(5), 938956. doi: 10.1037/0022-3514.92.5.938

Directions: Each question has two parts for you to complete. First, circle if you think the question is true or false. Then below your answer circle how sure you are of your answer. For example, if you're positive that you're correct circle "100\% absolutely." If you're just guessing circle " $50 \%$ just guessing."

1. The library catalog is the best place to go to search for academic journal articles. This statement is [ True / False ]

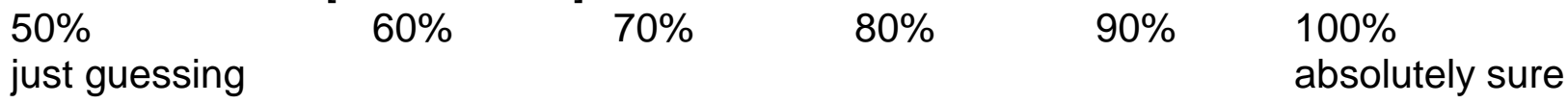

2. The following is an MLA citation for a book:

Tabery, J. (2014). Beyond versus: The struggle to understand the interaction of nature and nurture. Cambridge, MA, US: MIT Press.

This statement is [ True / False ]

$\begin{array}{lllll}50 \% & 60 \% & 70 \% & 80 \% & 90 \%\end{array}$

just guessing absolutely sure

3. An abstract is a summary of a journal article.

This statement is [ True / False ]

$\begin{array}{lllll}50 \% & 60 \% & 70 \% & 80 \% & 90 \%\end{array}$

just guessing absolutely sure

4. You need to use an in-text citation in a paper when you're directly quoting an author and when you're paraphrasing his/her words.

This statement is [ True / False ]

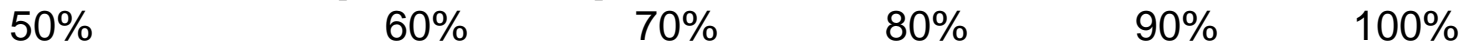

just guessing absolutely sure

5. If you were writing a paper on the rates of social anxiety among Facebook users and you wanted to find articles in a library database the best way to search would be to write your idea out as a sentence into the search box.

This statement is [ True / False ]

$60 \%$

$70 \%$

$80 \%$

$90 \%$

$100 \%$

just guessing

absolutely sure 
6. A newspaper article is not a peer-reviewed (scholarly) source. This statement is [ True / False ]

$50 \%$

$60 \%$ $70 \%$

$80 \%$

$90 \%$

$100 \%$

just guessing

absolutely sure

7. The journal title in the following article citation is Anthrozoös.

Curb, L. A., Abramson, C. I., Grice, J. W., \& Kennison, S. M. (2013). The relationship between personality match and pet satisfaction among dog owners. Anthrozoös, 26(3), 395-404. doi:10.2752/175303713X13697429463673

This statement is [ True / False ]

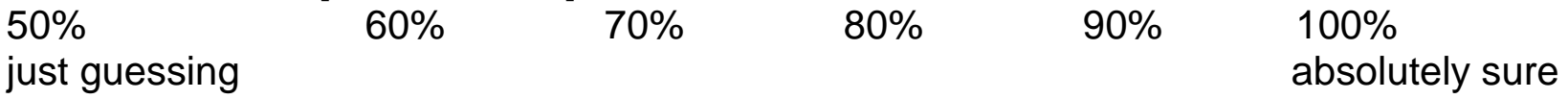

8. All of the articles in the online databases that the library subscribes to are also freely available on Google.

This statement is [ True / False ]

$50 \%$

$60 \%$

$70 \%$

$80 \%$

$90 \%$

$100 \%$

just guessing

absolutely sure

9. If the author of a book or article is dead you do not need to cite them in your paper.

This statement is [ True / False ]

$50 \%$

$60 \%$

$70 \%$

$80 \%$

$90 \%$

$100 \%$

just guessing

absolutely sure

10. SF446.5.M38 2002bx is an example of a call number.

This statement is [ True / False ]

$50 \%$

$60 \%$

$70 \%$

$80 \%$

$90 \%$

$100 \%$

just guessing

absolutely sure 


\section{Appendix B: General Knowledge Confidence Scale}

Age:

Gender:

Year in school:

Have you visited the library with a class for library instruction? Yes/No (Hint: These classes are usually held on the fifth floor of LLC)

Several of the questions are adapted from a scale developed by Michailova (2010). Citation: Michailova, J. (2010). Development of the overconfidence measurement instrument for the economic experiment. http://mpra.ub.unimuenchen.de/34478/1/MPRA paper 34478.pdf

Directions: Each question has two parts for you to complete. First, circle if you think the question is true or false. Then below your answer circle how sure you are of your answer. For example, if you're positive that you're correct circle "100\% absolutely." If you're just guessing circle "50\% just guessing."

1. An instant camera is still called a Polaroid.

This statement is [ True / False ]

$50 \%$

$60 \%$

$70 \%$

$80 \%$

$90 \%$

$100 \%$

just guessing

absolutely sure

2. Aphrodite is the Greek goddess of wisdom.

This statement is [ True / False ]

$50 \%$

$60 \%$

$70 \%$

$80 \%$

$90 \%$

$100 \%$

just guessing

absolutely sure

3. Microsoft is the company of Bill Gates.

This statement is [ True / False ]

$50 \%$

$60 \%$

$70 \%$

$80 \%$

$90 \%$

$100 \%$

just guessing

absolutely sure

4. Oslo is the capital of Norway.

This statement is [ True / False ]

$50 \%$

$60 \%$

$70 \%$

$80 \%$

$90 \%$

$100 \%$

just guessing

absolutely sure

5. Lake Huron, one of the five Great Lakes, is the largest lake in the world.

This statement is [ True / False ]

\begin{tabular}{|c|c|}
\hline $50 \%$ & $80 \%$ \\
\hline
\end{tabular}

6. Grand Central Terminal in New York City is the largest train station in the world. 
Partnership: The Canadian Journal of Library and Information Practice and Research, vol. 10, no. 2 (2015)

This statement is [ True / False ]

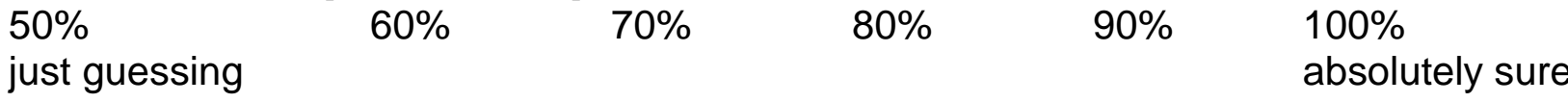

7. A spectroscope is the instrument used by people in a submarine to see objects above the water.

This statement is [ True / False ]

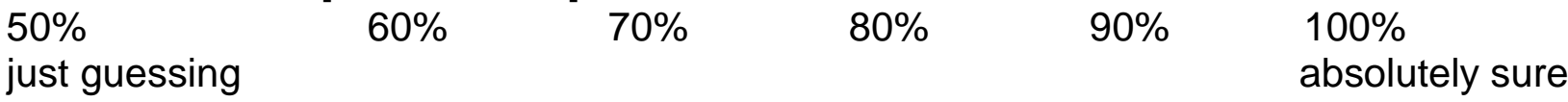

8. Vitamin C is a type of ascorbic acid.

This statement is [ True / False ]

$50 \% \quad 60 \% \quad 70 \%$

just guessing

$80 \% \quad 90 \% \quad 100 \%$

absolutely sure

9. "To be, or not to be, that is the question" is a quote from William Shakespeare's play Romeo and Juliet.

This statement is [ True / False ]

$\begin{array}{llllll}50 \% & 60 \% & 70 \% & 80 \% & 90 \% & 100 \%\end{array}$

just guessing absolutely sure

10. The 19th Amendment to the U.S. Constitution gave women the right to vote in elections.

This statement is [ True / False ]

$50 \%$

$60 \%$

$70 \%$

$80 \%$

$90 \%$

$100 \%$

just guessing

absolutely sure 